\title{
Color blend retrievals: Compromise memories or deliberate compromise responses?
}

\author{
ROBERT F. BELLI \\ University of New Hampshire, Durham, New Hampshire
}

\begin{abstract}
A total of 978 subjects, in two experiments and two pilot studies, were asked to recognize the color of objects to which they had been exposed in a slide presentation. Taken together, the studies demonstrate that an object's typical coloration influences recognitions, resulting in retrievals that compromise or blend the actual color and the typical color. In addition, Experiment 2 suggests that color recognitions may be simultaneously influenced by typical knowledge, event information, and postevent information. The findings question the adequacy of both the deliberate compromise and the compromise memory hypotheses as accounts for color blend retrievals. The deliberate compromise hypothesis fails to account for evidence that blend retrievals result because an intact representation of an event is not available at the time of test. The compromise memory hypothesis fails to account for evidence that deliberative processes do occur, and is in need of further exposition given that blend retrievals may be the result of different processes when resulting from typical knowledge and from postevent information. In conclusion, neither hypothesis, in isolation, is able to account for the various possible processes that appear responsible for blend retrievals.
\end{abstract}

In recent years, a considerable number of studies have investigated the influence of postevent information on subjects' retrievals of original event information (e.g., Bekerian \& Bowers, 1983; Loftus, 1975, 1977, 1979; Loftus, Miller, \& Burns, 1978; McCloskey \& Zaragoza, 1985a; Weinberg, Wadsworth, \& Baron, 1983). Some of this research has been concerned with understanding the nature of compromise retrievals. For example, Loftus (1977, Experiment 1) presented to subjects a series of slides that included one scene involving a green car. Following the slides, misleading postevent information was introduced to approximately half of the subjects that suggested that the car was blue; the remaining (control) subjects received no postevent color information. Upon retrieval, after a 20-min filler activity, subjects were asked to select one hue, from a series of hues, that most closely matched the color of the car. Control subjects tended to select accurate green hues, whereas misled subjects often

Pilot Study 1 was based on research conducted in partial fulfillment of the degree of Master of Arts in psychology at the State University of New York at Geneseo, and was presented at the 55th Annual Meeting of the Eastern Psychological Association, Baltimore, April 1984. I am grateful to several individuals who offered suggestions during the formative or later stages of the research and writing: Victor Benassi, George Rebok, Kenneth Kallio, Margaret Matlin, John Limber, and Karen Duffy. The thoughtful and challenging reviews provided by Michael McCloskey, Elizabeth Loftus, D. A. Bekerian, and an anonymous reviewer, on earlier submissions of the manuscript, were extremely helpful. Alice Healy also contributed substantive comments. Special gratitude is extended to Marcia Place, Bob Place, and Steve Sadlon, who voluntarily participated as the actors shown in the slide presentation, and also to Jessie La Cross, who helped with the collection of data. Requests for reprints should be addressed to Robert F. Belli, Department of Psychology, 134 Wesley Hall, Vanderbilt University, Nashville, TN 37240 . recognized the car as a green-blue blend, that is, as a compromise between the original event information and the postevent information. In related research, Loftus (1975, Experiment 2) found compromise retrievals also to result after the introduction of misleading postevent information that 4 or 12 persons participated in an event in which 8 persons had actually been depicted.

Loftus and her associates (Loftus, 1975, 1977, 1979; Loftus, Schooler, \& Wagenaar, 1985) interpreted these compromise retrievals as evidence for the existence of compromise memories. In this view, the memory of the original event information becomes altered with the introduction of the postevent information; that is, the postevent information is believed to "integrate" with the original event information, forming a single compromise memory for the event.

McCloskey and Zaragoza (1985a, 1985b) challenged the notion that compromise retrievals are reflections of compromise memories. In their view, compromise retrievals are the result of a deliberate compromise between the event and postevent informations that reside independently in memory. Specifically, the deliberate compromise hypothesis asserts the existence of two memorial representations at the time of retrieval: an intact memory of the original event information and an intact memory of the misleading postevent information. McCloskey and Zaragoza argued that although subjects are able to retrieve the original event information, they also remember having been informed (by the experimenter) otherwise. Since subjects have no reason to doubt that the postevent information provided by the experimenter is at least partially correct, they provide a compromise response. For example, subjects who remember having seen a green car, and also remember being informed that the car was blue, may 
reason that the color of the car could be described as being both green and blue, that is a green-blue blend. ${ }^{1}$

The present paper reports research that has implications with respect to the controversy of whether color blend retrievals result from compromise memories or from deliberate compromise responses. The two present experiments were motivated by the attempt to understand and extend the findings of two pilot studies. The results of Pilot Study 1 were noteworthy for the tendency of control subjects to select hues that were blends of green and yellow in remembering the color of an object that was predominantly green. Pilot Study 2 demonstrated that the object was not simply misperceived as being greenyellow in color. The findings of the pilot studies motivated research that supported the hypothesis that subjects' prior knowledge of an object's typical coloring influences their recognitions, leading to compromise retrievals between the original color and the typical color.

The strategy undertaken in this paper is to appeal to both the compromise memory hypothesis and the deliberate compromise hypothesis as potential explanations of color blend retrievals that result from the influence of prior knowledge. To the extent that each hypothesis adequately accounts for these findings, implications with respect to the postevent situation are drawn.

\section{PILOT STUDY 1}

Pilot Study 1 was conducted at the State University of New York at Geneseo, and was designed as an attempt to replicate Loftus's (1977, Study 2) finding of color blend retrievals resulting from the introduction of postevent information, with the use of a different critical object (i.e., something other than a car). Ninety-one subjects viewed a visual presentation of 20 color slides; the 10 th slide of the sequence depicted a young woman pouring a drink from a green plastic pitcher. The plastic pitcher was unobstructed and displayed near the center of the slide, against a white background. ${ }^{2}$ Following the slides, half the subjects were misled that the color of the pitcher was yellow (yellow condition), whereas the other half received no subsequent color information regarding the pitcher (control condition).

After an unrelated filler activity of $20 \mathrm{~min}$, the subjects were given a retrieval test. The subjects were presented a slide of an Ostwald color wheel, consisting of 24 hues of color (see Kuppers, 1973), and were asked to select the hue that most nearly matched the color of the pitcher. The hues on the Ostwald color wheel represent the visible spectrum ranging from $420 \mathrm{~nm}$ to $660 \mathrm{~nm}$ (Verity, 1980 ). Each hue was identified with an adjacent number. The numbers ranged from 1 to 24 , forming an ordinal scale of measurement based on wavelength. Numbers 1-5 were paired with purple hues (bluish red to reddish blue), 6-9 with blue (blue to greenish blue), 10-14 with green (bluish green to yellowish green), 15-19 with yellow (greenish yellow to yellowish orange), and 20-24 with red hues (reddish orange to red). The color of the plastic pitcher most closely matched hue \#12, a predominantly green hue.

To my surprise, the recognition responses of the subjects in the yellow and control conditions did not significantly differ, as tested by a Mann-Whitney $U$ test $(z=1.12$, one-tailed, $p=.13)$. The results were directional, however, with subjects in the yellow condition selecting hues more toward yellow $(M d n=15.23)$ than the hues selected by control subjects $(M d n=14.63)$. The responses of both groups were remarkable with respect to the frequent selection of hues that were blends of green and yellow (on the color wheel, hues $\# 13, \# 14$, and \#15). Specifically, $36.4 \%$ of the subjects in the yellow condition and $53.7 \%$ of the subjects in the control condition selected green-yellow hues.

Although subjects in the yellow condition were expected to have a high proportion of green-yellow retrievals, the tendency for such blend retrievals among control subjects was completely unexpected. One possible explanation for the performance of control subjects was that the color of the pitcher simply was misperceived. Pilot Study 2 tested recognition of the color of the plastic pitcher immediately after exposure. The subjects predominantly selected the closest matching green hue (\#12). The results of Pilot Study 2 demonstrate that the color blend retrievals among control subjects in Pilot Study 1 are not attributable to the pitcher's simply being misperceived as green-yellow.

\section{PILOT STUDY 2}

Pilot Study 2 involved the participation of 88 subjects from the University of New Hampshire. The subjects were shown 6 color slides, one at a time. Immediately following each slide, the subjects selected a hue, from the same Ostwald color wheel that was used in Pilot Study 1, that most closely matched an object shown in each slide. The 6 slides were selected from the series of 20 slides shown in Pilot Study 1. One of the slides depicted the green plastic pitcher. The other slides depicted different objects: two red candles, a blue jacket, a green sweater, a red bowl, and a purple sweatshirt. Each slide was presented for $4 \mathrm{sec}$. While viewing each slide, the subjects were told to focus on the object whose color they would be asked to recognize.

Following the color recognition responses to the six objects, all subjects were engaged in a color matching task. Each of the six slides was projected simultaneously with the color wheel slide, and the subjects were asked to match the color of the objects with a hue from the color wheel.

Of particular importance were the responses of subjects concerning the color of the plastic pitcher. With recognitions that occurred immediately following presentation of the slide, 65 of the 88 subjects ( $73.9 \%$ ) selected the green hue \#12, whereas the remaining 23 subjects $(26.1 \%)$ chose the yellowish green hue \#13. No other color selections were made. The matching data revealed a similar pattern of responses: 72 subjects $(81.8 \%)$ selected hue $\# 12$, and the remaining 16 subjects $(18.2 \%)$ chose hue $\# 13$. 
Of the 88 subjects, $69(78.4 \%)$ selected the same hue during matching as had been chosen during immediate recognition. The remaining 19 subjects had varied responses. Specifically, of the 65 subjects who initially selected hue \#12, 6 subjects $(9.2 \%)$ later matched the color of the pitcher with hue \#13. There was a more pronounced tendency among the 23 subjects who initially chose hue $\# 13$ to later match with hue $\# 12$, as $13(56.5 \%)$ of these subjects did.

The results demonstrate an overall preference for hue \#12 as best representing the actual color of the plastic pitcher. However, given that there also were substantial selections for the yellowish green hue $\# 13$ and, moreover, that at least some subjects shifted responses from hue \#12 during recognition to hue \#13 during matching, the findings suggest that although hue $\# 12$ most closely matches the color of the pitcher, the actual color lies somewhere between hues $\# 12$ and \#13. In addition, the fact that over half of the subjects who initially recognized the color of the pitcher with hue \#13 later matched with hue $\# 12$ provides further support that the color of the pitcher most closely resembles hue $\# 12$.

Although there were some subjects who recognized and perceived the pitcher with a yellowish green hue, the selections of hues that were blends of green and yellow among control subjects in Pilot Study 1 were more pronounced. In Pilot Study 1, there were more frequent selections of green and yellow blends. Also, the selections were more yellowish (e.g., there were many selections of hues \#14-\#16) in comparison with the selections of subjects in Pilot Study 2. From these results, the notion that control subjects in Pilot Study 1 simply misperceived the color of the green plastic pitcher as being yellowish does not adequately account for the data.

Another explanation of the yellowish selections of Pilot Study 1 involves the hypothesis that plastic pitchers are typically yellow, that subjects have knowledge to this effect, and that the subjects' retrievals of the pitcher were based on the blending of both the green event information and the yellow typical information.

\section{THE INFLUENCE OF KNOWLEDGE OF THE TYPICAL}

There is an extensive body of theory and evidence that proposes and demonstrates that everyday knowledge and understanding of what typically occurs will lead to inaccuracies during the remembering of a specific past event (e.g., Bartlett, 1932; Brewer \& Nakamura, 1984; Brewer \& Treyens, 1981; Carmichael, Hogan, \& Walter, 1932; Cohen, 1966; Crosland, 1921; Daniel, 1972; Friedman, 1979; Graesser, Woll, Kowalski, \& Smith, 1980; Minsky, 1975; Rumelhart \& Ortony, 1977; Schank \& Abelson, 1977). This literature, taken together, proposes that our knowledge of the typical exists as "default" assignments in memory. Friedman (1979), for example, discussed a typical kitchen as having the default assignments of a refrigerator, a stove, counters, and several small appli- ances, such as a toaster, a percolator, and a blender. During the remembering of a past event or situation, an individual may infer the existence of default assignments that, in actuality, were not present.

As an example, Brewer and Treyens (1981) found that subjects who experienced a 30-sec exposure to an academic office later remembered the office to contain books and windows, when in fact these items were not present. Books and windows are default assignments for a typical academic office. According to Brewer and Treyens, the nonpresent default assignments became integrated with other actual aspects (both typical and atypical) of the office during remembering.

An important finding among some researchers (i.e., Crosland, 1921; Daniel, 1972; Graesser et al., 1980) has been that the influence of typical knowledge increases in retrievals with increasing delay after initial exposure. Apparently, with very short retention intervals, subjects are generally able to accurately remember aspects of the event; with longer retention intervals, default assignments are more characteristic of memories. For example, Daniel (1972) exposed subjects to distorted figures of common animals. With recognition tests provided up to $5 \mathrm{~min}$ after exposure, subjects were accurate in selecting the figures they had seen earlier. With retention intervals of $20 \mathrm{~min}$ or more, however, subjects' recognitions were less distorted than were the original figures; that is, retrievals were compromised toward selection of those figures that were more representative of the typical outlines of animals.

The fact that compromise retrievals tend to result after some amount of delay is especially relevant for evaluating the deliberate compromise hypothesis as an explanation of Daniel's (1972) results. Subjects who are tested after a short delay are apparently aware of the distinction between their typical knowledge and what was experienced; with longer delays, such an awareness of this distinction apparently no longer exists, ruling out the possibility that the compromise retrievals are the result of a deliberate selection of stimuli that lie between typical knowledge and the actual event information.

Generalizing from this literature, the compromise green-yellow retrievals of subjects in the control condition in Pilot Study 1 might have been the result of an integration of the actual green color of the plastic pitcher, with the default assignment of yellow coloration in subjects' memories of plastic pitchers. Additional empirical work was conducted to test this hypothesis.

\section{OVERVIEW OF THE EXPERIMENTS}

In Experiment 1, plastic pitchers were shown to be typically considered as yellow in color. When procedures similar to those employed with the control subjects in $\mathrm{Pi}$ lot Study 1 were followed, subjects selected more yellowish hues with increases in the delay between exposure to the pitcher and retrieval. This finding is consistent with the literature, which has demonstrated that typical 
knowledge is more representative of retrievals with increasing retention intervals. In addition, Experiment 1 also provided more direct evidence that typical yellow coloring influences subjects' recognitions of the color of the green plastic pitcher as being more yellowish. The results of Experiment 1 seriously question the deliberate compromise hypothesis as an adequate explanation of compromise retrievals that result from the influence of typical knowledge. This limitation of the deliberate compromise hypothesis questions its adequacy as an explanation of compromise retrievals that result from the in troduction of postevent information. However, as discussed later, other aspects of the findings of Experiment 1 require that the compromise memory hypothesis also be evaluated.

Experiment 2 was motivated to compare the influences of postevent information that is either consistent or inconsistent with typical knowledge. Subjects were misinformed following exposure to the slides that the color of the pitcher was either yellow (consistent) or blue (inconsistent), or no color information was provided. Results were that recognitions by subjects misled with either color were shifted toward the postevent information in comparison with those of the control group. These findings have additional implications with respect to the deliberate compromise and the compromise memory hypotheses.

\section{EXPERIMENT 1}

\section{Method}

\section{Subjects}

Three hundred thirty introductory psychology students at the University of New Hampshire participated as subjects. The sample consisted of 120 males and 210 females. All subjects received course credit for their participation. The mean age for subjects was 19.8 years, and ranged from 17 to 55 years.

\section{Conditions and Procedures}

The subjects were tested in groups ranging from 9 to 30 , and participated in one of three conditions. Procedures for the control condition are described first. The subjects in the control condition followed almost the same procedures used with the control group in Pilot Study 1. There were five major phases to this procedure: viewing the slides, answering questions concerning the slides, participating in a 20 -min filler activity, taking a multiple-choice recognition test, and answering a final questionnaire on visual abilities and demographic characteristics. Before viewing the slides, the subjects were instructed that they would be shown "a series of slides that tell a story about three characters: a woman, Marcia, and two men, Bob and Steve," and that they should "pay close attention to the slide presentation."

The slides. The visual presentation consisted of 20 slides. A slide projector was programmed to present each slide for $4 \mathrm{sec}$ with a 1 -sec interval between slides.

The slide presentation opened with a slide of a young woman (Marcia) setting a table for two for dinner. During the time she is setting the table, two young men (Bob and Steve) in another apartment begin to share a bottle of whiskey. As time passes, Marcia clearly becomes discouraged because the person she is expecting for dinner has not arrived. Marcia begins to remove the dinnerware from the table. After the table has been cleared, the 10th slide in the sequence shows Marcia pouring herself a drink from the green plastic pitcher. The green plastic pitcher was seen only in this one slide. The sequence resumes with Bob and Steve continuing to drink while playing a game of chess. More time passes as Marcia broods while drinking and smoking. Finally, at a late hour, Bob is seen leaving the apartment where he was drinking with Steve. Bob enters the other apartment, whereby he encounters Marcia and an argument ensues.

The questions. Immediately after viewing the slides, all subjects answered a series of eight questions on what they had seen. Only one question referred to the plastic pitcher: "Do you think that the drink Marcia poured herself from the pitcher was alcoholic?" There was no postevent color information provided for any of the objects in the series of questions. The subjects responded to all eight questions by answering "yes" or "no."

The filler activity. After answering the series of eight questions, the subjects were engaged in a reading comprehension task for $20 \mathrm{~min}$. The subjects read paragraphs unrelated to all other experimental materials and were required to answer questions on these paragraphs.

The multiple-choice recognition test. Following the filler activity, the subjects were shown the same slide of an Ostwald color wheel that was used in Pilot Studies 1 and 2. During the projection of the color wheel, the subjects were asked to recognize the colors of six objects that were seen in the slides, including the plastic pitcher, candles, a vinyl jacket, a sweater, a plastic salad bowl, and a sweatshirt. The subjects were told to "indicate by number the color that most nearly matches the color of each object as it appeared in the slides." The dependent measure consisted of the numbers recorded by the subjects to indicate the recognized colors of these objects. The green plastic pitcher most closely matched hue \#12 on the color wheel slide.

The final questionnaire. Following the color recognition test, the subjects were queried about their visual abilities, including visual acuity and color vision. The subjects also provided information on sex, age, and whether they had been previously informed by anyone other than the experimenter of the intent of the experiment.

The experimental conditions. The results from the control condition were compared with the results from two other conditions. The subjects in the no-filler condition followed the same procedures as those in the control condition, except that they did not engage in a 20-min filler exercise. In the probable condition, the subjects participated in two sessions that were held 1 week apart. The procedure for session 2 of the probable condition was the same as for the single session of the control condition. During session 1 of the probable condition, the subjects were asked to indicate, without viewing any objects, a single most probable color for each of 18 objects. Of these 18 objects, one was "a plastic pitcher that would be used to store beverages." Also, the subjects selected probable colors for a candle, a vinyl jacket, a sweater, a plastic salad bowl, and a sweatshirt, among, of course, the other remaining objects. During the selection of most probable colors, a slide of the same Ostwald color wheel used in Pilot Studies 1 and 2 was projected. The subjects were instructed to choose only one number from the color wheel slide for each object.

\section{Results and Discussion}

Of the 330 subjects, the data from 25 subjects were omitted from analyses due to reported color weakness or blindness, acuity problems, or an apparent awareness of the intent of the experiment. The data from 105 subjects in the control group, 98 subjects in the no-filler group, and 102 subjects in the probable group were analyzed.

\section{Recognitions of the Pitcher}

To effectively demonstrate a yellow typicality effect for color recognitions of the green plastic pitcher, several 
results are desirable. Foremost, the most probable color of a plastic pitcher should be yellowish, according to the responses of subjects in the probable condition during session 1. Second, since subjects in the control group had a longer delay of $20 \mathrm{~min}$ between exposure to the pitcher and recognition, their responses were expected to be more toward the yellowish hues than the responses of subjects in the no-filler condition. Third, because inferences are to be made about typical coloring as an influence on the color recognitions in the control condition, the recognitions of the probable group during session 2 should not significantly differ from the responses of the control group. Fourth, recognitions of the color of the green plastic pitcher by the control and probable groups should be shifted toward yellow, as was the case in the responses of the control group in Pilot Study 1. Fifth, subjects in the probable condition who selected yellow hues as the most probable color of a plastic pitcher should have more yellowish recognitions than other subjects in the probable condition; to demonstrate a general typicality effect, subjects who selected blue hues as the probable color should have more bluish recognitions. Finally, to demonstrate that the recognitions of the color of the plastic pitcher constitute a blend of both prototypical yellow coloring and the actual green coloring of the pitcher, (1) the recognitions by subjects in session 2 of the probable condition should be significantly more toward the actual color of the pitcher than the probable color responses in session 1, and (2) green-yellow recognitions by individual subjects must be traceable to influences of both green event information and yellow typical knowledge.

Analyses have confirmed almost all of the above results. Table 1 shows the grouped relative frequencies of selections of the most probable color of a plastic pitcher (probable condition, session 1), as well as the recognitions of the color of the pitcher by the control, no-filler, and probable (session 2) conditions. First, note the responses of the probable group during session 1 . Clearly, most subjects chose yellowish hues $(M d n=16.08) ; 63.7 \%$ of responses were selections of hues \#15-\#19. Also there were few green and red responses, and very infrequent blue or purple responses. Apparently, people generally consider plastic pitchers to be yellowish, and less frequently consider them to be other colors.
As expected, in comparing the recognitions of subjects in the control and no-filler conditions, responses in the control condition were toward yellow in comparison with those in the no-filler condition. A Mann-Whitney $U$ test revealed that the difference in recognitions between the control $(M d n=14.09)$ and the no-filler $(M d n=13.31)$ conditions was directional, but not significant $(z=-1.23$, one-tailed, $p=.11$ ). The directionality of the results was more evident from an analysis on only those responses that were in the green to yellow range (hues $\# 10-\# 19$ ); control subjects' recognitions $(n=92, M d n=14$.14) were nearly significant as being more toward yellow than nofiller subjects' recognitions $(n=83, M d n=13.33)$, as revealed by a Mann-Whitney $U$ test $(z=-1.51$, one-tailed, $p=.07)$. The findings are consistent with other research (e.g., Crosland, 1921; Daniel, 1972; Graesser et al., 1980) that has shown that typical knowledge becomes more representative of retrievals with increasing delay. In the present study, typical knowledge of pitchers as yellowish was more influential in recognitions that occurred after an increased delay of $20 \mathrm{~min}$. Note, however, that many of the recognitions among subjects in the no-filler condition, who experienced a rather short retention interval, were also somewhat shifted away from green and toward yellow. There are a couple of possible explanations for this result. Perhaps the typicality influence was particularly strong with this particular situation, ${ }^{4}$ such that even with a short retention interval, the blending of information was already taking place. In addition, some subjects might not have remembered, even with the short retention interval, the actual color of the pitcher, and relied exclusively on their typical knowledge. Finally, some responses might have been influenced by the fact that the color of the pitcher was not exactly matched by hue \#12, leading to selections of hue \#13.

As shown in Table 1, a similar pattern was evident in the color recognitions of both the probable group (session $2, M d n=14.56)$ and the control group $(M d n=14.09$; recall that the color of the pitcher most closely matches hue \#12). Results of a Mann-Whitney $U$ test were nonsignificant $(z=0.73, p=.46)$, suggesting that session 1 for subjects in the probable group did not differentially influence their session 2 color recognitions of the pitcher in comparison with recognitions by control subjects. The

Table 1

Grouped Relative Frequencies (in \%) of Responses About Color of Plastic Pitcher by Control, No-Filler, and Probable Conditions in Experiment 1

\begin{tabular}{lccccc}
\hline & Hue & $\begin{array}{c}\text { Control } \\
\text { Condition }\end{array}$ & $\begin{array}{c}\text { No-Filler } \\
\text { Condition }\end{array}$ & \multicolumn{2}{c}{$\begin{array}{c}\text { Probable Condition } \\
\text { Session 1 }\end{array}$} \\
\hline Purple & $1-5$ & 4.8 & 8.2 & 4.9 & Session 2 \\
Blue & $6-8$ & 1.9 & - & 4.9 & 2.9 \\
Bluish Green & $9-11$ & 1.0 & 4.1 & 2.9 & 1.0 \\
Green & 12 & 14.3 & 20.4 & 6.9 & 17.6 \\
Yellowish Green & 13 & 21.9 & 21.4 & 2.9 & 16.7 \\
Yellowish Green & 14 & 10.5 & 4.1 & 2.0 & 7.8 \\
Greenish Yellow & 15 & 18.1 & 11.2 & 14.7 & 16.7 \\
Yellow & 16 & 5.7 & 14.3 & 18.7 & 6.9 \\
Yellowish Orange & $17-19$ & 16.2 & 9.2 & 30.4 & 14.7 \\
Red & $20-24$ & 5.7 & 7.1 & 11.8 & 12.7 \\
\hline
\end{tabular}


finding is consistent with a Mann-Whitney $U$ test performed on only those responses in the green to yellow range (hues \#10-\#19), because the recognitions of the probable group (session 2, $n=83, M d n=14.19$ ) did not significantly differ from those of the control group $(n=92$, $M d n=14.14$ ), with $z=-0.25, p=.80$.

Table 2 contrasts the grouped relative frequencies of session 2 recognitions of the green plastic pitcher by subjects in the probable group who selected yellowish hues (\#15-\#19, probable condition-yellow) and by subjects who selected other hues (probable condition-other) as the probable color of a plastic pitcher during session 1 . Clearly, recognitions by subjects in the probable-yellow condition $(n=65)$ are shifted more toward yellow $(M d n=15.06)$ than are recognitions by subjects in the probable-other condition ( $n=37, M d n=13.25)$. A MannWhitney $U$ test demonstrated that the difference in recognitions between these conditions is significant $(z=2.14$, one-tailed, $p \doteqdot .02$ ). It appears that subjects' typical knowledge concerning the color of plastic pitchers influenced their recognitions of the color of a green plastic pitcher that they had seen.

Although it was expected that subjects in the probable group who selected bluish hues (\#6- \#9) as the most probable color of a plastic pitcher should have recognitions shifted into the blue range, only 5 subjects selected bluish hues during session 1 , and none of their recognitions was bluish. These subjects, however, did have a tendency toward accurate recognition $(M d n=12.75)$. No firm conclusions can be drawn due to the small size of this sample.

So far the discussion has focused on results that demonstrate the influence of typical yellow coloring on the color recognitions of the green pitcher. In addition, the visual color information of the pitcher as green influenced recognitions. This result is best demonstrated by comparing the tendency of subjects in the probable condition during session 2 to recognize the pitcher as a blend of green and yellow ( $M d n=14.56)$, whereas their responses during session 1 centered on yellowish hues as the most probable colors $(M d n=16.08$, see Table 1). A Wilcoxon repeated measures test confirmed that the probable group's recognitions were significantly closer to the actual color of the

Table 2

Grouped Relative Frequencies (in \%) of Color Recognitions of Plastic Pitcher by Subjects in Probable Condition Who Selected Yellow or Other Colors as Most Probable Pitcher Color in Experiment 1

\begin{tabular}{|c|c|c|c|}
\hline \multirow[b]{2}{*}{ Color } & \multirow[b]{2}{*}{ Hue } & \multicolumn{2}{|c|}{ Probable Condition } \\
\hline & & Yellow & Other \\
\hline Purple & $1-5$ & 3.1 & 2.7 \\
\hline Blue & $6-8$ & 4.6 & - \\
\hline Bluish Green & $9-11$ & - & 2.7 \\
\hline Green & 12 & 13.8 & 24.3 \\
\hline Yellowish Green & 13 & 10.8 & 27.0 \\
\hline Yellowish Green & 14 & 6.2 & 10.8 \\
\hline Greenish Yellow & 15 & 21.5 & 8.1 \\
\hline Yellow & 16 & 7.7 & 5.4 \\
\hline Yellowish Orange & $17-19$ & 16.9 & 10.8 \\
\hline Red & $20-24$ & 15.4 & 8.1 \\
\hline
\end{tabular}

pitcher (hue \#12) than were their probable color responses $(z=3.06$, one-tailed, $p=.001)$. In fact, considering only those subjects in the probable group who selected yellow hues (\#15-\#19, probable-yellow condition) as the most probable color of a pitcher, the comparison of their session 1 responses $(M d n=16.40)$ with their recognition responses $(M d n=15.04$, see Table 2$)$ was revealed by a Wilcoxon repeated measures test to also be significant $(z=2.61$, one-tailed, $p<.01)$.

Results also demonstrate the tendency of subjects to recognize the color of the pitcher as a green-yellow blend, a finding consistent with the blend retrievals of subjects in the control group in Pilot Study 1. In Experiment 1, $41.2 \%$ of subjects in the probable condition and $50.5 \%$ of subjects in the control condition selected hues that were green-yellow blends (i.e., hues \#13-\#15).

Of the 65 subjects in the probable-yellow condition, 25 $(38.4 \%)$ selected hues that were green-yellow blends (hues \#13-\#15) during recognition. Only 4 of these responses can be accounted for by subjects who selected hue \#15 both as the probable color of a pitcher and as the recognized color of the pitcher they had seen. With the probable-yellow condition, it appears that subjects who were selecting green-yellow hues were doing so as a compromise between green event information and yellow typical knowledge.

Surprisingly, of the 37 subjects in the probable-other condition, a substantial number $(N=17,45.9 \%)$ also selected hues \#13-\#15. There are various possibilities that could explain this result. One possibility is that since subjects had been asked for only one response as the most probable color, a yellow hue would have been the likely second choice. Especially for those subjects who selected hues unrelated to green (e.g., purple or red), typical yellow information might have influenced their responses. Admittedly, however, the result might have been due to some effect not related to typical coloration at all. As we have seen from Pilot Study 2, some subjects upon immediate testing and matching selected hue \#13. The greenyellow retrievals among subjects in the probable-other condition may simply be an extension of this tendency.

However, given that there may be a tendency to select green-yellow hues that is unrelated to any influence of typical coloration, there is still evidence supporting a yellow typicality effect. Analyzing only the recognitions that were blends of green and yellow (hues \#13-\#15), a MannWhitney $U$ test revealed that the recognitions among subjects in the probable-yellow condition $(M d n=14.61)$ were significantly more toward yellow than the recognitions of those in the probable-other condition $(M d n=13.35$ ) $(z=2.42$, one-tailed, $p<.01)$. Removing the responses of the 4 subjects who had selected hue \#15 during both session 1 and session 2 (there were no duplicates of selections of hues \#13 or \#14), the recognitions of subjects in the probable-yellow condition were still significantly more yellowish $(N=21, M d n=14.38)$ than those of subjects in the probable-other condition: as demonstrated by a MannWhitney $U$ test, $z=1.88$, one-tailed, corrected $p<.05$. 
These results suggest that although the probable-other subjects might have tended to recognize the color of the pitcher as yellower than it actually was due to some influence other than typical coloration, the responses of the probable-yellow subjects reflected an influence of yellow typical knowledge that worked in addition to any other tendency to remember the pitcher as yellowish.

Taken together, the results of Experiment 1 reveal an overall tendency for color recognitions of the pitcher to be influenced by both green event information and typical knowledge of pitchers as yellow. Furthermore, a substantial portion of responses resulted from an actual compromise of these two sources of information.

\section{Recognitions of Other Objects}

The color recognitions of the other five objects were generally accurate. Because each of these objects except the bowl was shown in more than one slide, the tendency for accuracy is not surprising. The color of the plastic bowl may have been well remembered because the bowl appeared in the last slide of the sequence and served as the focal point of a dramatic event (Marcia hit Bob in the head with the bowl). Table 3 lists the median responses as to color of objects by subjects in the single sessions of the control and no-filler conditions and in each session of the probable condition. In addition, the number of slides or exposures in which each object appeared and the hue that each object most closely matches are listed in Table 3. Mann-Whitney $U$ tests comparing the color recognitions of each of these five objects by subjects in the probable and control conditions never approached significance (absolute values of $z \mathrm{~s}<0.76, p \mathrm{~s}\rangle .44$ ), suggesting no differential color recognitions among probable and control groups.

Inasmuch as most of the objects were presented in a number of slides, any influence of typical coloring would suggest a rather robust effect. In a comparison of recognitions by subjects in the control and no-filler conditions, Table 3 reveals that the recognitions toward two objects, the candles and the sweater, were directional, with responses of control subjects being more toward typical coloring than responses of subjects in the no-filler condition. However, as revealed by Mann-Whitney $U$ tests, only the color recognitions of the candles by control and no-filler groups approached significance $(z=-1.08$, onetailed, $p=.14$ ). A comparison of the color recognitions of the sweater between the two conditions resulted in $z=0.47$, one-tailed, $p=.32$.
However, analyses of the responses of the subjects in the probable condition demonstrate that the color recognitions of two of the objects, the candles and the jacket, were significantly influenced by typical coloration. A Mann-Whitney $U$ test comparing color recognitions of the red candles by subjects in the probable condition who selected red hues $(\$ 20-\$ 24)$ as the most probable color of candles $(N=40, M d n=21.98)$ with those by subjects who selected yellow hues $(\# 15-\# 19)$ as the most probable color of candles $(N=51, M d n=21.25)$ resulted in $z=2.66, p<.01$. The color recognitions of the blue jacket by subjects selecting green or yellow hues $(\# 10-\# 19)$ as the most probable color of a vinyl jacket $(N=21, M d n=8.88)$ significantly differed from those by subjects selecting purple hues (\#1-\#5) as the most probable color $(N=24, M d n=6.50)$, as revealed by a MannWhitney $U$ test $(z=2.10$, one-tailed, $p=.02)$. These results suggest that typical coloration may generally influence the memory of the colors of viewed objects, and that the effect is not isolated to a limited number of objects.

\section{Competing Hypotheses}

Typical coloration exists as knowledge that subjects may bring into an experimental situation. The tendency of subjects to select green-yellow blends as the color of the pitcher apparently resulted from a compromise between two sources of information: yellow typical coloration and the actual green information supplied by the event itself. Two competing hypotheses attempt to explain the compromise retrievals in the postevent situation: the compromise memory hypothesis of Loftus and her associates (Loftus, 1977, 1979; Loftus et al., 1985) and the deliberate compromise hypothesis of McCloskey and Zaragoza (1985a, 1985b). Both of these hypotheses are evaluated here as potential explanations of the color blend retrievals that were demonstrated to be influenced by typical coloration in Experiment 1. In addition, by means of generalization, the adequacy of each hypothesis in accounting for blend retrievals that occur in the postevent situation is also discussed.

The deliberate compromise hypothesis. McCloskey and Zaragoza (1985a, 1985b) argued that in the postevent situation subjects are able to access intact and independent representations of both the event and postevent informations. Although subjects accurately remember the event information, they deliberately select a compromise response between the event information and the postevent information as they reason that their memories of both

Table 3

Median Responses to Colors of Five Objects in Experiment 1

\begin{tabular}{lccrrrrr}
\hline & $\begin{array}{c}\text { Number of } \\
\text { Object }\end{array}$ & $\begin{array}{c}\text { Matching } \\
\text { Exposures }\end{array}$ & $\begin{array}{c}\text { Control } \\
\text { Hue }\end{array}$ & $\begin{array}{c}\text { No-Filler } \\
\text { Condition }\end{array}$ & \multicolumn{2}{c}{ Condition } & \multicolumn{2}{c}{ Probable Condition } \\
\cline { 7 - 8 } Candles & 3 & $22(\mathrm{red})$ & 21.55 & 21.85 & 17.05 & 21.73 \\
Jacket & 6 & 8 (blue) & 8.29 & 8.21 & 8.02 & 8.00 \\
Sweater & 4 & 11 (green) & 12.05 & 12.28 & 6.79 & 11.61 \\
Bowl & 1 & 21 (red) & 21.70 & 21.27 & 11.44 & 21.59 \\
Sweatshirt & 12 & 5(purple) & 6.60 & 6.69 & 7.58 & 6.53 \\
\hline
\end{tabular}


sources of information must be at least partially correct. In sum, the deliberate compromise hypothesis assumes that an intact representation of the original event information is accessible at the time of retrieval.

This assumption is untenable in consideration of the green-yellow blend retrievals among subjects in Experiment 1 . If subjects were able to accurately remember the pitcher as green, on what basis would they deliberately compromise this memory with their knowledge that plastic pitchers are typically yellow? It is not unreasonable to assume that subjects can become conscious of their knowledge of typical coloration. Minsky (1975) suggested that typical default knowledge can arise to conscious awareness; perhaps, for example, subjects during retrieval conjure up an image of their yellow plastic pitcher that they have at home. However, given that subjects are able to access an intact representation of the green pitcher, why would subjects conjure up a default representation in the first place, and, in the second, deliberately choose a compromise of both sources of information? Apparently, any compromising would result precisely because an intact green representation is not accessible at the time of retrieval.

Clearly, for blend retrievals to occur, actual event information must exist in memory, perhaps in some indistinct state. The literature (e.g., Crosland, 1921; Graesser et al., 1980; Rumelhart \& Ortony, 1977) offers a model of the processes that occur when typical default knowledge integrates with event information. As suggested by the recognitions of subjects in the no-filler condition, with shorter retention intervals there is a greater likelihood for a relatively intact representation of the event to be accessible, and fairly accurate retrievals will result. With increasing delay, however, the representation of the event information becomes more indistinct (either through fading or growing inaccessibility). During remembering, then, subjects are (in some sense) uncertain whether the green information that they have in memory is accurate, and their typical knowledge is used to "reconstruct" the content of what was experienced. Color blend retrievals, then, can be far less deliberate than what is suggested by the hypothesis of McCloskey and Zaragoza (1985a, 1985b).

The reconstruction model proposes that the extent of the influence of typical knowledge on remembering depends on the status of the event information in memory. For subjects who have no memory for the event information, either because such information was never encoded or because the event information was "lost" with the passage of time, retrievals will tend to be based solely on typical knowledge. For subjects who have an indistinct memory for the event information, blend retrievals are likely to result. Finally, subjects with intact representations of the event information will be accurate during remembering.

As argued, blend retrievals occur in the direction of typical knowledge because an intact representation of event information is not available at the time of retrieval.
There is no reason to assume that intact representations of event information have a greater chance of being accessed in the postevent situation than the extent suggested by the occurrence of typical blends. At the very least, the lack of an intact representation of event information leads to a very reasonable explanation of blend retrievals that result as a compromise of event and postevent informations: subjects use any available postevent information to reconstruct, during retrieval, the content of their experience. Compared with subjects who received postevent misinformation, control subjects (who are not misled) tend to be accurate, even if their event representations are indistinct. Control subjects have no conflicting postevent information on which to base their retrievals. In a real sense then, postevent information can influence the remembering of event information, an assertion that McCloskey and Zaragoza (1985a, 1985b) disputed.

Compromise memory hypothesis. According to the compromise memory hypothesis, a blend retrieval is a reflection of a single integrated memory to which both event and postevent informations contribute (Loftus, 1975, 1977; Loftus et al., 1985). If both sources of information are equally influential, the compromise memories should tend to blend together both sources of information to an equivalent extent. For example, if the event information is green, and the postevent information is blue, compromise memories would be expected to consist of blends that are $50 \%$ green and $50 \%$ blue. If either of the two sources of information is more influential than the other, then the compromise memories should have a higher percentage of the more influential source of information.

The results of Experiment 1 show quite a different pattern. As Tables 1 and 2 illustrate, the green-yellow hue \#14 was less preferred than either the green-yellow hue \#13 or the yellow-green hue \#15. Also, hue \#14 was infrequently selected in both conditions of Pilot Study 1 and in all three conditions of Experiment 2 (see below), in comparison with hues $\# 13$ and \#15. Apparently, hue \#14 is an unattractive alternative, and this finding questions the adequacy of the compromise memory hypothesis to account for the data.

Although I have sought to adequately account for the infrequent selection of hue \#14, I have not been able to do so. ${ }^{5}$ However, speculation can reveal reasons why certain blends do not occur. Consider subjects who are shown a yellow apple, and are later misled (or have typical knowledge) that the apple is red. ${ }^{6}$ Would subjects represent the apple as orange? Orange apples are implausible; thus, at the very least, compromise memories are limited by certain constraints. Moreover, preventing implausible compromise memories may involve some sort of deliberative process. Apparently, in the present series of studies, some constraint limited the selection of hue \#14.

Another situation in which compromise memories would appear to be implausible involves the remembering of items. For example, in previous studies (e.g., 
Bekerian \& Bowers, 1983; Loftus et al., 1978), subjects were exposed to a yield sign and then were provided misleading postevent information that instead there was a stop sign. It would seem quite implausible for subjects to form a compromise memory that was an integration of a stop sign and a yield sign. However, Weinberg et al. (1983), by using a yellow yield sign as event information and a stop sign as the misleading postevent information, found that when subjects were provided a test in which they were to select between yellow and red yield signs as the sign shown in the event, misled subjects significantly more often selected the red yield sign than did control subjects.

Loftus et al. (1985) argued that the compromise retrievals of selecting the red yield sign in the Weinberg et al. (1983) study demonstrated the existence of a compromise memory that "reflects features of both the original and postevent information" (Loftus et al., p. 378). However, it is rather straining to believe that subjects spontaneously integrated features of a yellow yield sign and a red stop sign into some compromise memory that could be a close match to the red yield sign shown during the recognition test. More likely, as McCloskey and Zaragoza (1985a, 1985b) suggested, the compromise retrievals found in the study by Weinberg et al. are the result of processes of deliberation.

One reasonable possibility offered by McCloskey and Zaragoza (1985a) to account for Weinberg et al.'s (1983) compromise retrievals simply assumes that a proportion of misled subjects did not remember the yellow yield sign, but did remember being informed of the existence of the stop sign. These subjects selected the red yield sign because of its closer similarity to a stop sign. Another possibility is that subjects had intact representations of both the event and postevent informations, and selected the red yield sign as a deliberate compromise between these two sources of information. In addition, a reconstruction model interpretation (see above) suggests a third possibility. Misled subjects might have had an indistinct representation of the yellow yield sign, and used the postevent information (perhaps also in some indistinct state) to help in deciding between the alternatives on the test. Their tendency would then have been to select the red yield sign.

Both the deliberate compromise hypothesis of McCloskey and Zaragoza (1985a, 1985b) and the reconstruction memory model (e.g., Bartlett, 1932; Graesser et al., 1980; Neisser, 1967; Rumelhart \& Ortony, 1977) assert a possible role for deliberation. However, a distinction exists between these two views with respect to specifying the conditions that will lead to deliberation. In McCloskey and Zaragoza's view, deliberation will occur even if an intact representation of the event is available at the time of remembering. With the reconstruction memory model, any deliberation will occur precisely because an intact event representation is not available; rather, the representation is conceived as being indistinct or "fragmented." According to the reconstruction memory model, an intact representation needs only to be reflected during remembering, whereas a fragmentary representation requires the rememberer to inferentially use sources of information other than the event (e.g., typical knowledge, and perhaps postevent information) to reclarify the experience.

In the attempt to account for compromise retrievals that occur with discrete features of experiences, such as items (e.g., Weinberg et al., 1983), a reasonable interpretation favors processes of deliberation at the expense of any pure compromise memory account. On the other hand, it is quite possible that compromise memories that do not primarily involve deliberation may at times be responsible for blend retrievals of continuous features of experience, such as color (Loftus, 1977) or number (Loftus, 1975; see also Loftus et al., 1985). However, in the final analysis, the compromise blend notion must acknowledge, to a greater extent than previously indicated, the considerable importance that processes of deliberation may assume with influences of postevent information.

\section{Motivation for Additional Research}

The results of Experiment 1 suggest that typical coloration influences the remembering of the colors of objects; this finding has implications with respect to hypotheses concerning the influence of postevent information on remembering. In Experiment 2 postevent information concerning the color of the pitcher was introduced to determine whether misinformation that is consistent or inconsistent with typical coloration would influence color recognitions. Pilot Study 1 already provided some indication that postevent information that is consistent with yellow typical coloration led to a greater shift in responses toward yellow, although the responses of subjects misled with yellow did not significantly differ from those of control subjects. I reasoned that increasing the power of the analysis by using a larger sample size might lead to a significant difference. In addition, introducing a condition in which subjects receive postevent misinformation that is inconsistent with typical coloration (i.e., blue misinformation) might further elucidate the respective influences that postevent information and typical coloration have on the remembering of event information.

\section{EXPERIMENT 2}

\section{Method}

\section{Subjects}

Four hundred sixty-nine students enrolled in introductory psychology courses at the University of New Hampshire participated as subjects. All subjects received course credit for their participation. The sample consisted of 180 males and 289 females. The mean age was 18.5 years and ranged from 17 to 28 years.

\section{Procedure}

The subjects were tested in sessions ranging from 24 to 34 subjects in size. All aspects of the procedure were basically the same as in 
Pilot Study 1 and Experiment 1. The subjects were shown the same series of slides that included the slide with the green plastic pitcher. Immediately following the slides, the subjects were asked questions on certain aspects of what they had seen; in these questions, the postevent misinformation was introduced.

In the questions immediately following the slides, approximately one-third of the subjects received yellow postevent misinformation concerning the color of the pitcher (yellow condition), one-third received blue misinformation (blue condition), and one-third received no misinformation (control condition). The fourth question for subjects in the yellow condition was, "Do you think that the drink Marcia poured herself from the yellow pitcher was alcoholic?' 'For the blue condition, the word "blue' appeared in place of the word "yellow." For subjects in the control condition, neither the word "yellow" nor the word "blue" appeared. Also, in the series of questions, the colors of three other objects were accurately provided. The subjects answered the questions by responding "yes" or "no."

Following a 20-min filler activity, the subjects recognized the colors of six objects by selecting hues on the same Ostwald color wheel slide used in the previous studies. Recognitions included the color of the pitcher. Before being dismissed, the subjects provided demographic information, assessed their visual abilities, and indicated whether they had been informed of the intent of the study before their participation.

\section{Results and Discussion}

The responses of 67 subjects were omitted from analyses due to reported visual problems or an apparent awareness of the intent of the experiment. In addition, 3 subjects were eliminated because they did not respond to the question about the color of the pitcher. Omissions resulted in the control, yellow, and blue conditions consisting of 132, 136 , and 131 subjects, respectively.

Table 4 shows the grouped relative frequency distribution for the three conditions. Table 4 shows that in comparison with responses in the control condition, the responses of subjects in the blue condition were more toward blue, and the responses of subjects in the yellow condition were more yellowish.

Subjects in both the yellow and control conditions tended to select inaccurate yellowish hues (control $M d n=14.28$; yellow $M d n=15.24$ ), whereas subjects in the blue condition tended to select accurate green hues (blue $M d n=12.00$ ). The results of an overall KruskalWallis test are significant $\left[\chi^{2}(2, N=399)=60.34\right.$, $p<.001]$. Furthermore, planned Mann-Whitney $U$ com-

Table 4

Grouped Relative Frequencies (in \%) of Color Recognitions of the Pitcher in Experiment 2

\begin{tabular}{lcrcc}
\hline & & \multicolumn{3}{c}{ Condition } \\
\cline { 3 - 5 } Color & Hue & Blue & Control & Yellow \\
\hline Purple & $1-5$ & 7.6 & 5.3 & 2.2 \\
Blue & $6-8$ & 20.6 & 1.5 & - \\
Bluish Green & $9-11$ & 9.9 & 1.5 & 3.7 \\
Green & 12 & 23.7 & 17.4 & 10.3 \\
Yellowish Green & 13 & 8.4 & 18.2 & 10.3 \\
Yellowish Green & 14 & 5.3 & 6.8 & 6.6 \\
Greenish Yellow & 15 & 9.2 & 23.5 & 22.8 \\
Yellow & 16 & 5.3 & 2.3 & 25.7 \\
Yellowish Orange & $17-19$ & 6.9 & 12.9 & 16.9 \\
Red & $20-24$ & 3.1 & 10.6 & 1.5 \\
\hline
\end{tabular}

parisons show that responses in both misled conditions differed significantly from those in the control condition: comparing the yellow condition with the control condition results in $z=2.21$, one-tailed, $p<.02$; comparing the blue condition with the control condition results in $z=-5.63$, one-tailed, $p<.0001$. Although subjects in both yellow and control conditions tended to select hues that were blends of green and yellow (see Table 4), the yellow condition subjects selected more yellowish hues than did the control subjects. Also, subjects in the blue condition more frequently selected bluish hues than did control subjects. Specifically, subjects in the blue condition selected a smaller percentage of green-yellow hues than did control subjects and selected a higher percentage of bluish green and blue hues than did control subjects (see Table 4).

Table 4 reveals that subjects in the blue condition slightly more often selected hue \#12, the closest matching hue to the color of the pitcher, than did control subjects. Using a more liberal measure of accuracy to also include the two hues adjacent to hue \#12 (\#11 and \#13), equivalent percentages of subjects in the control and the blue conditions selected these accurate green hues (36.4\% and $35.9 \%$, respectively). This finding suggests that equivalent percentages of subjects in both conditions may have had good memories for the color of the pitcher and were not influenced by typical coloration or by any postevent misinformation to which they may have been exposed. Perhaps only those subjects who had a very poor memory (or no memory) for the event information were influenced by sources extraneous to the event. Thus, the tendency of subjects in the blue condition to have more bluish retrievals than those of control subjects might not have been due to any graduated shift in responses; rather, some proportion of blue condition subjects-who, if control subjects, would have selected inaccurate yellowish hues-may have accepted the postevent information and selected inaccurate bluish hues.

Although this latter possibility cannot be ruled out as an explanation of the findings for the blue condition, the responses of the subjects in the yellow condition suggest that the misinformation resulted in a graduated shift in responses, even among subjects who (as controls) would have demonstrated an accurate memory for the event. Using the liberal measure of accuracy, the yellow condition subjects chose accurate green hues significantly less frequently (23.5\%) than did subjects in the other two conditions $\left[\chi^{2}(2, N=399)=6.54, p<.05\right]$. If there are no extraneous influences on initial memory, there is no apparent reason for the accuracy performance of subjects in the yellow condition to be poorer than that of subjects in the control condition.

Thus, at the very least, the introduction of postevent misinformation, when consistent with typical expectation, does appear to result in a graduated shift in recognition responses. Due to the influence of postevent information, yellow condition subjects, who would have selected accurate green hues if they had not been misled, had a ten- 
dency to select green-yellow hues; those who would have (without having been misled) selected green-yellow hues, tended to select even more yellowish hues.

Given that the responses of subjects in the yellow condition are suggestive of the occurrence of shifting color recognition, the responses of subjects in the blue condition may also have been shifted in comparison with those of controls. That is, the introduction of postevent blue misinformation may have caused subjects who would have, as controls, selected accurate green hues to select bluish hues, and those subjects who would have selected green-yellow hues, as controls, to be influenced toward choosing green hues.

\section{Competing Hypotheses}

The findings of Experiment 2 support the notion that color retrievals can be influenced by various combinations of three sources of information: event information, postevent information, and typical coloration. The results lead to considerations of the adequacy of both deliberate compromise and compromise memory hypotheses as accounts for the data.

The deliberate compromise hypothesis. As discussed earlier, typical coloration appears to influence retrievals only if an intact pristine representation of event information is not available. On the other hand, the deliberate compromise hypothesis (McCloskey \& Zaragoza, 1985a, $1985 \mathrm{~b}$ ) proposes that, with the introduction of postevent information, even subjects who have available an intact representation of the event information may tend to make a compromise response. In fact, deliberate compromises may occur in the postevent situation, although their occurrence is untenable in the situation in which typical knowledge influences retrievals. That is, there may be different processes responsible for the occurrence of blend retrievals in these two situations.

However, because the results of Experiment 2 suggest that both typical coloration and postevent information can work in combination to influence retrievals, for the deliberate compromise hypothesis to maintain its appeal, there must be some accounting for the simultaneous existence of both intact and nonintact event representations. Perhaps some subjects have an intact representation of the event and make a deliberate compromise in the direction of the postevent misinformation. In addition, however, other subjects may not have an intact representation. These latter subjects are quite likely to use either their typical knowledge, the postevent information, or both sources of information to reconstruct the experience. That is, although the deliberate compromise hypothesis is not entirely ruled out as an explanation of blend retrievals, the current data suggest quite strongly that other processes may be at work. At least for some subjects, it is quite likely that the introduction of postevent information does influence the ability to remember the event.

The compromise memory hypothesis. The fact that two sources of information-typical expectation and post- event information-lead to compromise retrievals also poses difficulties for the compromise memory hypothesis. These two sources of information appear to have a similar influence on responses, but may involve different processes. For example, compromising in the direction of typical knowledge may be a function of the growing indistinctiveness of the memory of the event with the passage of time and a reconstruction of the experience at the time of test. On the other hand, compromises that occur due to the influence of postevent information may be the result of deliberation at the moment the misinformation is introduced, with a subsequent loss of distinct memories of event and postevent informations at the time of test. If there do exist such different processes, then the distinguishing characteristics of two different types of compromise memories are in need of exposition.

\section{GENERAL DISCUSSION}

Taken together, the studies presented in this paper demonstrate that in the remembrances of a past event, color blend retrievals can result from the influences of both typical coloration and postevent color information. Although the influence of postevent information on color memory has been previously reported, the finding that typical coloration also influences the memory for the colors of objects encountered during a specific event is a major contribution of this paper. In addition, two hypotheses that have been offered as explanations of blend retrievals, the deliberate compromise hypothesis (McCloskey \& Zaragoza, 1985a, 1985b) and the compromise memory hypothesis (Loftus, 1975, 1977; Loftus et al., 1985), have been evaluated with respect to their adequacy in accounting for blend retrievals that involve the influences of typical coloration and postevent information.

There are difficulties associated with both hypotheses. The deliberate compromise hypothesis, which hinges upon the notion that an intact pristine representation of event information is available at the time of retrieval, is discredited, given the understanding that blend retrievals of color event information with typical coloration will likely result only when an intact event representation is not accessible. Also, there is reason to believe that intact event representations are not always available at the time of remembering by misled subjects who have made blend retrievals in postevent information experiments.

The compromise memory hypothesis suggests a blending mechanism that is spontaneous and unconscious. Evidence from the present series of studies indicates that processes of deliberation are operative to some extent in influencing blend retrievals, to the detriment of the compromise memory hypothesis. In addition, the compromise memory notion tends to be vague. To account for color blend retrievals that can arise from the influences of two sources-that is, typical coloration and postevent color information-the compromise memory hypothesis may 
need to be modified so as to distinguish between potential different processes that these two different sources may activate.

The present studies do not rule out the possibility that deliberate compromises can be responsible for some of the color blend retrievals between event and postevent informations. However, the deliberate compromise hypothesis is seriously questioned as providing a totally adequate explanation of blend retrievals. As outlined in this paper, there are other possibilities. For example, subjects may not have an intact memory of the event at the time of test and may use the postevent information to reconstruct the event. Also, subjects may spontaneously and unconsciously blend the two sources of information in memory at some point during retention or retrieval. In fact, given that there are individual differences among subjects, in any one experiment some or all of these possibilities may be responsible for blend retrievals.

Only through additional research can these various possibilities be adequately evaluated. For example, the deliberate compromise hypothesis would be enhanced by evidence that subjects can be induced to retrieve on the basis of intact event representations in an experiment in which compromising would likely occur. In addition, the relative merits of both the deliberate compromise and the compromise memory hypotheses may be further elucidated by experimental manipulation of the duration of intervals between exposure to the event, the introduction of postevent information, and the testing of memory.

The processes that are responsible for blend retrievals are potentially numerous and very involved. Their complexity deserves considerable appreciation.

\section{REFERENCES}

BARTLETT, F. C. (1932). Remembering: A study in experimental and social psychology. Cambridge: Cambridge University Press.

BekERIAN, D. A., \& BowERs, J. M. (1983). Eyewitness testimony: Were we misled? Journal of Experimental Psychology: Learning, Memory, \& Cognition, 9, 139-145.

Brewer, W. F., \& Nakamura, G. V. (1984). The nature and functions of schemas. In R. S. Wyer \& T. K. Srull (Eds.), Handbook of social cognition (Vol. 1, pp. 119-160). Hillsdale, NJ: Erlbaum.

Brewer, W. F., \& TREYENS, J. C. (1981). Role of schemata in memory for places. Cognitive Psychology, 13, 207-230.

Carmichael, L., Hogan, H. P., Walter, A. A. (1932). An experimental study of the effect of language on the reproduction of visually perceived form. Joumal of Experimental Psychology, 15, 73-86.

CoHen, R. L. (1966). Effect of verbal labels on recall of a visually perceived simple figure: Recognition vs. reproduction. Perceptual \& Motor Skills, 23, 859-862.

Crosland, H. R. (1921). A qualitative analysis of the process of forgetting. Psychological Monographs, 29(Whole No. 130).

DANIEL, T. C. (1972). Nature of the effect of verbal labels on recognition memory for form. Journal of Experimental Psychology, 96, 152-157.

Friedman, A. (1979). Framing pictures: The role of knowledge in automized encoding and memory for gist. Joumal of Experimental Psychology: General, 108, 316-355.

Graesser, A. C., Woll, S. B., Kowalski, D. J., \& Smith, D. A.
(1980). Memory for typical and atypical actions in scripted activities. Journal of Experimental Psychology: Human Leaming \& Memory, 6, 503-515.

Heison, H., \& LanSFord, T. (1970). The role of spectral energy of source and background color in the pleasantness of object colors. Applied Optics, 9, 1513-1562.

Kuppers, H. (1973). Color: Origin, systems, uses (F. Bradley, Trans.) London: Van Nostrand Reinhold.

LoFTUS, E. F. (1975). Leading questions and the eyewitness report. Cognitive Psychology, 7, 560-572.

LofTus, E. F. (1977). Shifting human color memory. Memory \& Cognition, $5,696-699$.

LofTus, E. F. (1979). Eyewitness testimony. Cambridge: Harvard University Press.

LofTus, E. F., Miller, D. G., \& Burns, H. J. (1978). Semantic integration of verbal information into a visual memory. Journal of Experimental Psychology: Human Learning \& Memory, 4, 19-31.

Loftus, E. F., Schooler, J. W., \& WagenaAR, W. A. (1985). The fate of memory: Comment on McCloskey and Zaragoza. Journal of Experimental Psychology: General, 114, 375-380.

MCCloskey, M., \& ZARAGOZA, M. (1985a). Misleading postevent information and memory for events: Arguments and evidence against memory impairment hypotheses. Journal of Experimental Psychology: General, 114, 1-16.

McCloskey, M., \& Zaragoza, M. (1985b). Postevent information and memory: Reply to Loftus, Schooler, and Wagenaar. Journal of Experimental Psychology: General, 114, 381-387.

MiNSKY, M. (1975). A framework for representing knowledge. In P. H Winston (Ed.), The psychology of computer vision (pp. 211-277). New York: McGraw-Hill.

NeIsSer, U. (1967). Cognitive psychology. New York: AppletonCentury-Crofts.

Rumelhart, D. E., ORTony, A. (1977). The representation of knowledge in memory. In R. C. Anderson, R. J. Spiro, \& W. E. Montague (Eds.), Schooling and the acquisition of knowledge (pp. $99-$ 135). Hillsdale, NJ: Erlbaum.

SCHANK, R., ABELSON, R. (1977). Scripts, plans, goals and understanding: An inquiry into human knowledge structures. Hillsdale, NJ: Erlbaum.

VERITY, E. (1980). Color observed. New York: Van Nostrand Reinhold. Weinderg, H. I., Wadsworth, J., \& Baron, R. S. (1983). Demand and the impact of leading questions on eyewitness testimony. Memory \& Cognition, 11, 101-104.

\section{NOTES}

1. The deliberate compromise hypothesis, as discussed in the text, is less appealing as an explanation of Loftus's (1975) findings with number. A subject who is shown 8 people in a videotape and is later misinformed that the number of people was 12 , is unable to compromise on the basis that there exists a number that could be described as being both 8 and 12 . However, a subject may determine (for whatever reason) that the postevent information is only partially correct (and thus determine that his/her own memory is only partially correct as well). In addition, a subject, upon remembering both event and postevent information, may be able to infer the experimenter's hypothesis and select a compromise value as a demand characteristic.

2. Black-and-white photocopies of the slide are available from the author upon request.

3. The selection of yellowish hues as the most probable color of a plastic pitcher is not a result confined to students at the University of New Hampshire. Similar results were found from the responses of 42 students at the State University of New York at Geneseo, in which $76.2 \%$ selected yellowish hues $(\# 15-\# 19, M d n=16.19)$.

4. One may note that Daniel (1972) did not find a typicality effect after a 5-min retention interval, whereas with the no-filler condition in Experiment 1, there was a typicality effect with a shorter delay (approximately $90 \mathrm{sec}$ ). The different acquisition conditions may be at least 
partially responsible for this discrepancy. In Daniel's study, subjects were shown only 1 slide, depicting only the stimulus figure, for $4 \mathrm{sec}$. With the no-filler condition in the present experiment, the green plastic pitcher was shown in 1 critical slide among 19 other slides, each for $4 \mathrm{sec}$. In addition, in the critical slide, there were other items in addition to the pitcher. Thus, the stimulus figure in Daniel's study was, in all likelihood, better encoded than the plastic pitcher in the no-filler condition, perhaps leading to a "stronger" memory that would take a longer time to lose its distinctiveness.

5. One possible explanation was that hue \#14 was unpleasant in color, and that its unpleasantness led to its lack of selection. In previous research, Helson and Lansford (1970) discovered that certain greenyellow hues are nonpleasing. In having subjects rate the pleasantness of hues on the color wheel, I found that all green-yellow hues (\#13-\#15) were equivalently unpleasant, and less pleasant than the predominantly green $(\# 12)$ and yellow $(\# 16)$ hues.

6. I am indebted to Michael McCloskey for this example.

(Manuscript received May 10, 1985; revision accepted for publication December $7,1987$. 